OAI-PMH: http://www.indteca.com/ojs/index.php/Revista Scientific/oai

Artículo Original / Original Article

\title{
Rol del maestro en los procesos de innovación educativa
}

Autor: Oscar Alfredo Rojas Carrasco Universidad Miguel de Cervantes, UMC oscar.rojas@umcervantesecontinua.cl

Talca, Chile

http://orcid.org/0000-0002-6739-5559

\section{Resumen}

El artículo se refiere al rol del maestro en el proceso de innovación educativa y su aporte al momento de modificar las prácticas docentes frente a los cambios tecnológicos. Con base en estudios sobre los procesos de los cambios y avances tecnológicos se discute de forma teórica el papel del maestro basado en la opinión de varios expertos y contrasta rasgos de diferentes autores y puntos de vista. Enfatiza la desarticulación de la innovación educativa con la institución y la organización que regulan el funcionamiento de las escuelas, así como la relevancia de la estructura de las prácticas docentes. La metodología es contrastar la opinión de los autores citados para concluir que el maestro debe integrase de forma activa y aplicar los procesos de Innovación Educativa en las aulas de clase.

Palabras clave: maestro; innovación educacional; tecnología.

Cómo citar este artículo:

Rojas, O. (2019). Rol del maestro en los procesos de innovación educativa. Revista Scientific, 4(Ed. Esp.), 54-67, e-ISSN: 2542-2987. Recuperado de: https://doi.org/10.29394/Scientific.issn.25422987.2019.4.E. 3.54-67

Fecha de Recepción:

03-05-2019
Fecha de Aceptación: 10-07-2019
Fecha de Publicación: 05-09-2019 


\section{Role of the teacher in the processes of educational innovation}

\begin{tabular}{|c|c|}
\hline 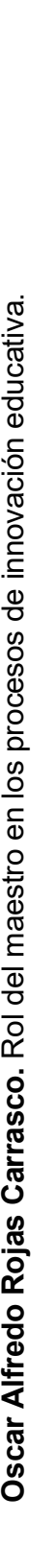 & $\begin{array}{l}\text { Abstract } \\
\text { The article refers to the role of the teacher in the process of educational } \\
\text { innovation and its contribution when modifying teaching practices in the face of } \\
\text { technological changes. Based on studies on the processes of technological } \\
\text { changes and advances, the role of the teacher is theoretically discussed based } \\
\text { on the opinion of several experts and contrasts traits of different authors and } \\
\text { points of view. Emphasizes the disarticulation of educational innovation with } \\
\text { the institution and organization that regulate the operation of schools, as well } \\
\text { as the relevance of the structure of teaching practices. The methodology is to } \\
\text { contrast the opinion of the cited authors to conclude that the teacher must be } \\
\text { actively integrated and apply the processes of Educational Innovation in } \\
\text { classrooms. }\end{array}$ \\
\hline & $\begin{array}{l}\text { How to cite this article: } \\
\text { Rojas, O. (2019). Role of the teacher in the processes of educational innovation. Revista Scientific, } \\
\text { 4(Ed. Esp.), 54-67, e-ISSN: } 2542-2987 \text {. Recovered from: https://doi.org/10.29394/Scientific.issn.2542- } \\
\text { 2987.2019.4.E.3.54-67 }\end{array}$ \\
\hline & $\begin{array}{ccc}\text { Date Received: } & \text { Date Acceptance: } & \text { Date Publication: } \\
03-05-2019 & 10-07-2019 & 05-09-2019\end{array}$ \\
\hline
\end{tabular}




\section{Introducción}

La innovación educacional, con el paso de los años viene a ser uno de los fundamentos del modelo que emplean las escuelas, por lo tanto, desde inicio de los años noventa las prácticas docentes vienen a ser impulsadas por este poderoso medio que permite inducir transformaciones en el sistema educativo conocido hasta ese momento. A partir de allí, algunos investigadores se han dado a la tarea de indagar en relación al impacto que estos cambios traen en todos los entornos escolares y medir su probabilidad de éxito y arraigo en las escuelas.

Ahora bien, los cambios son provocados y proponen afectar a gran parte de las escuelas de la zona, lo que corresponde con la resistencia a la aplicación de las nuevas tecnologías en los estudiantes, docentes, apoderados y directivos.

De igual manera, González (2014), señala que:

La educación necesita docentes con actitud emprendedora e innovadora, capaces de generar nuevos talentos, líderes que motiven a otros a actuar. Su principal papel es comunicar y enseñar nuevos conocimientos, para que los estudiantes o profesionales que se están formando, logren poseer las herramientas necesarias, tantos sociales como competencias técnicas, las cuales serán útiles para ser aplicadas en la vida laboral y personal de cada uno (pág. 68).

En Latinoamérica, la investigación ha sido un tema cuyo interés se ha acrecentado con el paso de los años, en cambio, los países desarrollados ya poseen grupos consolidados de investigación que les ha permitido generar cambios significativos en las instituciones a las que pertenecen. Toda investigación, permite construir cambios educativos coadyuvando a formular políticas propias de la reconstrucción de dichos métodos en las escuelas.

A partir de la investigación, se pueden obtener pistas para encontrar soluciones a la difícil tarea de hacer que el maestro ocupe su rol protagónico 
en la aplicación y uso de las innovaciones tecnológicas, una vez conocidas las orientaciones, se elabora el método de trabajo que proponga el cambio de los parámetros existentes y que permitan hacer propuestas innovadoras.

De acuerdo a los comentarios anteriores se propone, el cambio en la capacidad de investigación, la capacidad de inventiva y una aptitud que permita el cumplimiento de la planificación. Asimismo, se puede decir, que esta labor no es individualista, más bien se debe llevar a cabo con un equipo que permita la generación de ideas innovadoras y constancia para el logro de los objetivos y el cumplimiento de las metas de las instituciones educativas.

En el presente artículo se expone de forma teórica el papel del maestro basado en la opinión de varios expertos y contrasta rasgos de diferentes autores y puntos de vista que permitan finalmente llegar a conclusiones y aportes a todos los interesados dentro y fuera del entorno educativo.

Considerando que el objetivo es interpretar desde una óptica introspectiva el significado procesos de innovación educativa: se hace necesario describir la metódica empleada para llevarlas de principio a fin. La metodología se orientó inicialmente por una revisión documental de definiciones de grupos de investigación por diversos autores en Latinoamérica, luego del análisis e interpretaciones a estos conceptos, se presenta una definición, emergente que surge desde la interpretación introspectiva basada en la vinculación social que ocurre los procesos de innovación educativa.

Por tal motivo, metodológicamente incluye la exploración en la experiencia del autor como principales sujetos informantes. De allí que en una fase cumbre del escrito se otorga la profundidad al análisis de los elementos que dan sostenibilidad en el tiempo y finalmente se generan unas reflexiones finales donde se refleja la importancia que reviste el Grupo de Investigación como un método o vía para producir, gestionar el conocimiento y el liderazgo de los actores dentro de la modalidad educativa a distancia, en la cual se deben atender elementos distintivos que caracterizan para a partir de esa 
valoración se puede definir el potencial que contemplan como espacios epistémicos para el desarrollo de valores comunes, intereses, metas y proyectos académicos, fortalecer el talento humano que hace vida en la universidad y contribuir con la creación intelectual a cubrir necesidades de diversos contextos de la comunidad.

En ese sentido, el enfoque es interpretativo, socio-crítico y autorreflexivo, del proceso innovador por parte del docente como ente motivador para la formación de una cultura científica colaborativa, invita a superar errores cognitivos y mitos culturales que dentro del proceso educativo rompe las barreras y parcelas disciplinares para compartir y a partir del otro $u$ otros hacer ciencia.

Según Huberman (1983), citado por Escudero (1988):

El rol del profesor y su preparación profesional es decisiva en la realización del cambio, pero también goza de su propia entidad el papel del grupo de profesores, la función de los directores escolares, las funciones de apoyo de servicios externos y su organización a nivel regional o provincial. Sólo una adecuada complementariedad entre todas esas instancias y sujetos parece ofrecer algunas garantías más verosímiles para el éxito de proyectos innovadores (pág. 4).

Dicho autor indica que, si los estudiantes no adquieren los conocimientos, se debe reforzar y cambiar el proceso de enseñanza para lograr el objetivo propuesto. En este sentido, el rol fundamental lo posee es el maestro, quien tiene la tarea de generar espacios de renovación de conocimientos.

En consecuencia, Pérez (2004), citado por Arrieche (2018), afirma que:

Cuando se habla de innovación educativa, esta supone una serie de transformaciones en todos los ámbitos, por ello sostiene que: Innovar no solo implica hacerlo desde el ambiente, sino transformar a las personas desde sus propias concepciones de aprender y de conocer, darse cuente que la innovación se da en las estructuras cognitivas y en la 
concepción que se tiene del aprendizaje en relación a los sujetos concebir a los sujetos que aprenden desde perspectivas distintas en el aprendizaje, permite innovar las prácticas educativas (pág. 367).

\section{Retos de la Educación}

2.1. Generado por la globalización, la globalización, es un proceso pluridimensional, el cual contiene algunas dimensiones como la económica, la financiera, tecnológica y de comunicaciones, considerando aspectos como la educación, cultura y política, entre otros aspectos, para dar valor y calidad a la enseñanza e investigación, beneficiando conjunto de docentes ante la generación de cambios tecnológicos, desarrollando estrategias de innovación educativa.

2.2. Generado por la naturaleza del conocimiento, en la sociedad contemporánea la importancia fundamental la tiene el conocimiento y el manejo eficiente de la información, debido a que es la herramienta más eficaz para combatir la obsolescencia. En este sentido, es relevante generar disciplinas que incrementen la calidad del de carácter transdisciplinario.

Siguiendo con los argumentos que permitieron darle respuesta al planteamiento inicial se debe hacer referencia al rol del maestro en los procesos de cambio de la educación, ya que con el paso de los años se incrementan las críticas como por ejemplo: cuando un individuo no encuentra trabajo, permanecerá la mayor parte del tiempo en la calle, vinculándose con pandillas, y más aún si se encuentra en sectores vulnerables, es por ello que es fundamental que las escuelas proporcionen una enseñanza que se fundamente en los valores, y a su vez le proporcionen enseñanza académica de calidad.

Desde esta perspectiva, se puede decir que la calidad de un maestro es un camino al interior del individuo, por ende, lo más significativo es la práctica del individuo, sus reflexiones y la necesidad de mejorar, los buenos 
maestros, asisten puntualmente a sus clases, acomodan sus clases con antelación, tienen un estilo activo de enseñanza, revisan las actividades y dan cuenta de los resultados a los apoderados y son el principal apoyo en la enseñanza.

Por lo antes señalado, se puede afirmar que el maestro debe conocer y estar claro del papel clave y protagónico de acompañamiento que tiene sobre los alumnos y debe estar familiarizado con el cambio de paradigma educacional, donde el rol del maestro no es solo la comprobación del aprendizaje sino la enseñanza de la aplicación de los mismos en la sociedad.

Partiendo de la premisa que la innovación es denominada como un proceso que de manera premeditada y coordinada se estructura para darse de diversas formas, en este contexto, le teoría de la innovación realiza aportes significativos y puede ocurrir según diversos modelos, al respecto parafraseando lo planteado por Havelock y Huberman (1980), presentan tres de ellos: el modelo de proceso que comprende la resolución de investigación; el modelo de proceso de interacción social; y el modelo de proceso de resolución de problemas (págs. 15-380).

\subsection{Modelo de Proceso de Innovación según Havelock y Huberman}

2.3.1. El modelo de proceso que comprende la resolución de investigación, evidencia el modelo como una serie de fases en las cuales el proceso de innovación no se analiza desde la perspectiva del usuario (elemento pasivo); tampoco muestra la exploración como un conglomerado de réplicas especificas a diversos problemas, sino que más bien es un conjunto de ideas que experimentan transformaciones para generar productos útiles para el desarrollo de las instituciones, su personal y el proceso de enseñanza y aprendizaje. En este modelo el conocimiento de produce de forma masiva y se difunde a todo aquel a quien le pueda ser de utilidad, logrando la innovación.

El mencionado proceso se presenta a través de un modelo con enfoque 
lógico y racional de la innovación, sostenido en algunos supuestos que, si bien son cuestionables, pueden ser aplicados con un proceso de planificación bien definido que permita la vinculación de la teoría y la práctica.

2.3.2. El modelo de proceso de interacción social (humanista), comprende la difusión de la innovación y da relevancia a las redes interpersonales de información, liderazgo (transformacional), opinión y otros aspectos. En el modelo mencionado se centra la atención en la interacción entre los miembros del grupo adoptante, aquí la innovación hace que los participantes tomen conciencia y precisen un proceso de búsqueda de información que le sea de utilidad para su propia situación y evaluación.

2.3.3. El modelo de proceso de resolución de problemas, inicialmente parte tomando como eje central al usuario, y que existe la necesidad de innovar, ya dada esa hipótesis, se genera un diagnóstico, la aplicación y evaluación. Una vez consideradas las variables mencionadas se proponen posibles soluciones sobre estrategias de ejecución de los procesos centrada en actividades colaborativas con un enfoque participativo que no sea manipulable. Para éste, la asesoría externa corresponde con un punto de partida que permite establecer posibles soluciones que deben ser interiorizadas por los participantes y que respondan a las necesidades reales de los usuarios.

\section{Metodología}

En esta perspectiva la exigencia de una nueva racionalidad parece estar marcando el desarrollo de nuevos tiempos, y las cada vez más desarrolladas tecnologías parecen exigir nuevas formas de percibir, entender y comprender la realidad (fenomenología), así como nuevos contenidos del saber que con pertinencia para una forma de vida cada vez más alejada del esquema de organización socio-cotidiano al cual estamos acostumbrados. Se trata de un mundo que está en pleno proceso de transformación y que requiere de una concienzuda y serena interpretación (hermenéutica). 
De acuerdo a la interpretación del investigador se sustentó en categorías: Pedagogía humanista, liderazgo, estudiante, educación superior asumiéndose una configuración imaginaria de la experiencia vivida por los actores sociales vinculado a la academia en el saber docente.

\section{Resultados}

Después de haber procesado y analizado toda la información que emergió de este proceso heurístico, la etapa siguiente está representada por la construcción teórica, constituye el aporte sustantivo que hace el investigador cualitativo.

El rol del pedagogo humanista, implica que los alumnos aprendan y estos se conviertan en el docente una cuestión de inspiración mediante el ejemplo, en vez de imposición y obediencia. En cuanto a la pedagogía humanista parte de la valoración personal, el ser humano es el centro de todo, busca el bienestar, fortalece las relaciones humanas y transmite los valores humanos mediante una formación ciudadana en un contexto de espiritualidad con visión de futuro. Se desarrolla en el currículum integral a través de la transversalidad de los contenidos relacionando el desarrollo personal con el profesional, por medio de la orientación integral, el autodesarrollo, la inteligencia emocional, el manejo y resolución de conflicto, las técnicas de estudios, y el servicio comunitario.

En cierto modo es una realidad intangible, es como un arte escénico, un encuentro, es algo que se experimenta con la interacción del líder con otros seres humanos.

En función de todo lo expuesto en estas líneas, construyo teóricamente la conceptualización del estudiante como el centro de la educación y el sentido de todo accionar pedagógico, el cual exige la calidad educativa de los docentes para poder desarrollar un aprendizaje constante. Éstos se comprometen con las metas obteniendo un crecimiento personal que 
consolida su formación producto de la madurez.

Desde el punto de vista de la universidad, se busca reformar la propuesta formativa ya que sigue siendo rígida, caduca, trasnochada, y en ella, las unidades de enseñanzas se encuentran estáticas, mientras que los planes de estudio son muy especializados y la formación aparece de una forma fragmentada. Todas estas circunstancias han hecho que las casas de estudio se encuentren en una encrucijada de la desconfianza que inspira en el seno de la sociedad. Sin embargo, de las acciones depende que esa imagen tan negativa se pueda cambiar a una mucho más positiva, en la que se instaure otro tipo de estructuras, metodologías, proyectos, que recuperen la confianza en las instituciones educativas de nivel superior.

Figura 1. Representación gráfica de la construcción teórica.

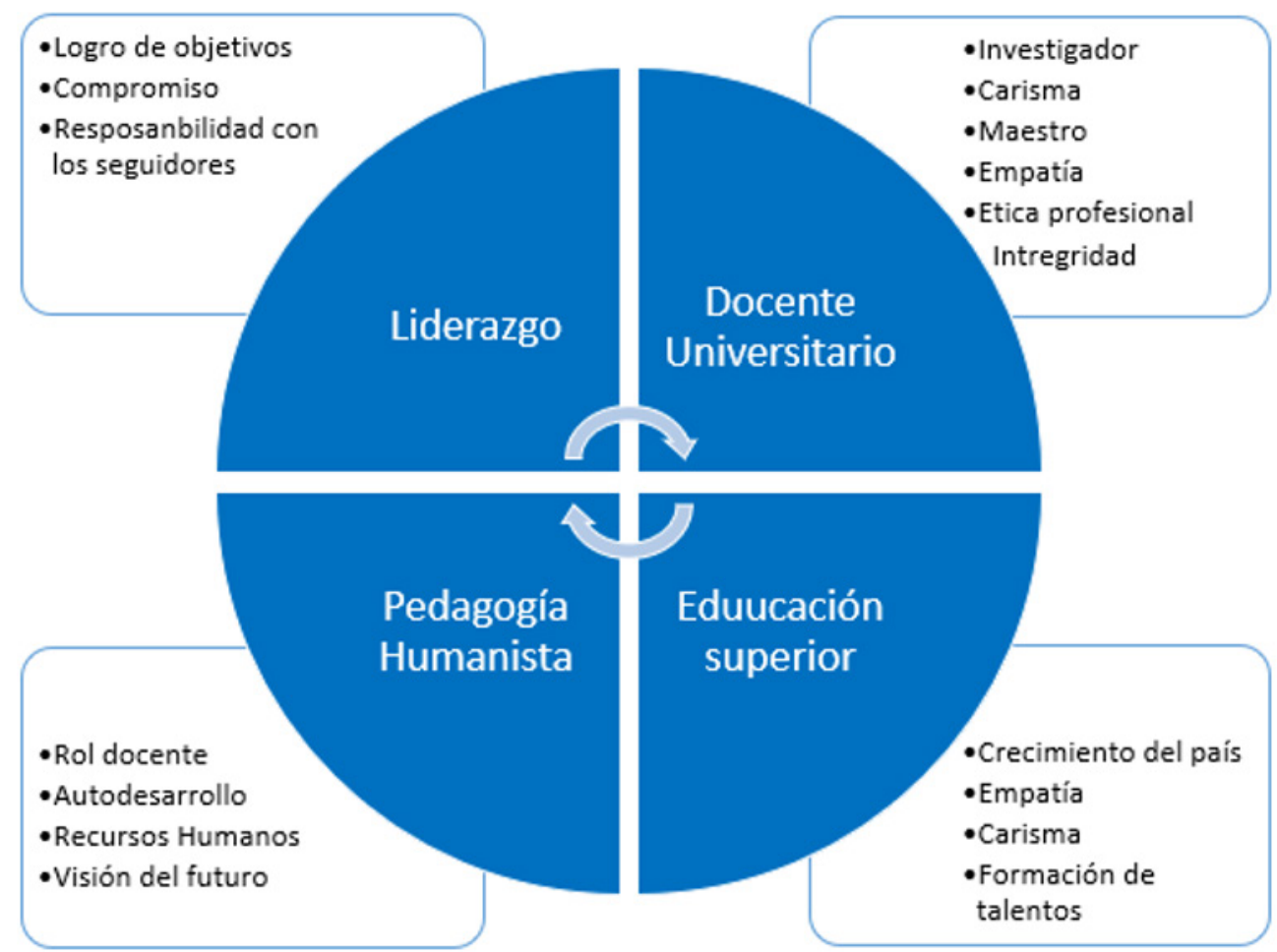

Fuente: El Autor (2019). 
En cuanto al acercamiento teórica de la educación superior, se infiere al sistema donde se estudia una carrera profesional y se obtiene una titulación, para el desarrollo de la sociedad donde se encuentra inmerso. Es un proceso educativo donde interviene la relación docente-alumno en un mundo de conocimientos y aprendizaje permanente. En comparación a los otros niveles de educación permanente, alcanza un estatus educativo mediante el crecimiento pedagógico del docente, para la preparación de sus estudiantes al mercado laboral, fortaleciendo la superación personal y el desarrollo como ser humano, como se muestra en la figura 1.

\section{Conclusiones}

El docente es elemento esencial para propiciar el proceso educativo, donde se ha incorporado la tecnología, por ello, se invita al lector a la aplicación de nuevas tecnologías, no de forma arbitraria, sino mediante la renovación del proceso educativo para hacerlo más eficaz y que dé respuesta a los cambios impuestos por una sociedad que está conformada por individuos sometidos a procesos evolutivos, mediante el uso de redes de información y diferentes elementos a través de canales informales.

Por tanto, la ampliación de los mercados requiere de una educación que ayude a las personas a realizar tareas para las cuales no fueron formadas pero para las cuales son aptas dadas las capacidades adquiridas mediante la praxis y que les permite prepararse para una vida profesional con una mejor aptitud para trabajar en equipo, a utilizar la información de manera autónoma y a desarrollar la improvisación como un elemento creativo que le permita tener un pensamiento complejo y aplicarlo dentro y fuera del contexto educativo.

Tomando como base lo anterior, se puede decir que una sociedad globalizada cada día impone retos de mayor envergadura en cuanto a la innovación en los procesos educativos, un docente innovador es capaz de transformarse y hacer que los estudiantes participen de forma creativa en sus 
aulas de clases. Conforme la bibliografía consultada, todo el entorno educativo debe reconocer el papel estratégico del maestro en la educación es vital ya que es uno de los pilares que tiene la formación de los futuros profesionales del país y en la generación y transmisión del conocimiento.

El artículo se finaliza interpretando según Barraza (2006a), que:

La innovación es un proceso organizado y creativo que parte de una necesidad que el profesor visualiza en su práctica y que trata de trascenderla para llegar a un cambio que impacte y que se debe evaluar para ver si se logró (pág. 5).

Continuando con el discurso de Barraza (2006b), indica que: la innovación no se inicia desde el aislamiento, ya que es un proceso donde se involucra la cooperación permanente como fuente de contraste y que fomenta el intercambio profesional, ya que la cooperación y el acuerdo general, darán mejores resultados que una estrecha supervisión, tomando en cuenta que la innovación se caracteriza por ser una actividad que implica cambios en actividades y actitudes dirigidas por un sistema innovador que sigue una sola dirección de verticalidad, dado que las propuestas generalmente vienen de fuera y deben ser aplicadas al interior de las instituciones por un profesorado que no las diseño (págs. 2-15); en este sentido, la recomendación es que los procesos de innovación educativa, sean generados y propuestos por los directivos, docentes, apoderados y estudiantes de los centros educativos, ellos garantizarán que la aplicación genere frutos efectivos, que cumplan con las necesidades de las mismas.

\section{Referencias}

Arrieche, M. (2018). Gestión Docente en el Contexto de la Educación Primaria Venezolana. Revista Scientific, 3(7), 354-373, e-ISSN: 25422987. Recuperado de: https://doi.org/10.29394/Scientific.issn.2542$\underline{2987.2018 .3 .7 .18 .354-373}$ 
Barraza, A. (2006a,b). Innovación Didáctica en Educación Superior: un estudio de caso. Diálogos educativos, (12), 2-15, e-ISSN: 0718-1310. Recuperado de:

https://dialnet.unirioja.es/servlet/articulo?codigo $=2473879$

Escudero, J. (1988). La innovación y la organización escolar. Localizado en: La gestión educativa ante la innovación y el cambio, ISBN: 84-2770829-7. España: Editores Narcea. Recuperado de:

http://www.terras.edu.ar/biblioteca/17/17GSTN Escudero Unidad 3.p df

González, T. (2014). La importancia de la Innovación y el Emprendimiento en los docentes del Sistema Educacional Chileno. Aspectos a considerar en la reflexión. Revista Electrónica Gestión de las Personas y Tecnología, 7(19), 68-78, e-ISSN: 0718-5693. Recuperado de: http://www.redalyc.org/articulo.oa?id=477847107006

Havelock, R., \& Huberman, A. (1980). Innovación y problemas de la educación: teoría y realidad en los países en desarrollo. Edición española, ISBN: 92-3-301527-0. París, Francia: Organización de las Naciones Unidas para la Educación, la Ciencia y la Cultura. Recuperado de: https://unesdoc.unesco.org/ark:/48223/pf0000136018 


\section{Oscar Alfredo Rojas Carrasco \\ e-mail: oscar.rojas@umcervantesecontinua.cl}

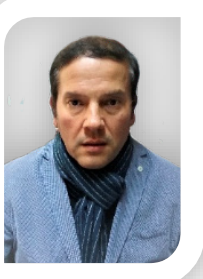

Nací en Chile, el 29 de noviembre del año 1974. Actualmente me desempeño como Académico Investigador para la Dirección de Postgrado e Investigación de la Universidad Miguel de Cervantes (UMC); Mis estudios académicos son de Post-Doctor in Finance, Doctor of Science in Economics and Finance de la Atlantic International University (AIU), Hawai, Estados Unidos; Doctorando en Educación de la Universidad Privada de Tacna (UPT), Perú; Máster Business Administration (MBA) de la Universidad de Talca (UTalca), Chile; Magíster en Educación de la Universidad Autónoma de Chile (UA); Diplomado en Administración de Políticas Públicas de la Universidad de Viña del Mar (UVM); Diplomado en Administración de Salud de la Universidad de Viña del Mar; Ingeniero Comercial de la Universidad de Talca; y Licenciado en Ciencias en la Administración de Empresas de la Universidad de Talca, en Chile. He publicado en Europa, 13 libros, principalmente, en Madrid, España, poseo múltiples publicaciones indexadas en WOS, SCOPUS, SciELO, y LATINDEX, entre otras; como también he dictado conferencias en congresos, tales como: "Propuesta Metodológica para la determinación del Coeficiente Beta de una empresa listada. Bases técnicas para el cálculo del valor razonable y su revelación bajo IFRS", de la Conferencia Interamericana de Contabilidad; "La globalización y sus repercusiones en Chile"; "Las redes sociales y su influencia en la educación" en el Encuentro Iberoamericano de Educación (EIDE); entre otras. 